\title{
MINIMAL RELATIVE RELATION MODULES OF FINITE $p$-GROUPS
}

\author{
MOHAMMAD YAMIN
}

(Communicated by Maurice Auslander)

\begin{abstract}
Consider $1 \rightarrow S \rightarrow E \rightarrow G \rightarrow 1$, where $G$ is a finite $p$-group generated by $g_{i}, 1 \leq i \leq d$, and $E$ a free product of cyclic groups $\left\langle g_{i}\right\rangle$, $1 \leq i \leq d$. If $d$ is the minimum number of generators for $G$, then we prove that the largest elementary abelian $p$-quotient $S / S^{\prime} S^{p}$, regarded as an $\mathbb{F}_{p} G$-module via conjugation in $E$, is nonprojective and indecomposable.
\end{abstract} sider

The author [5] has introduced and studied relative relation modules. Con-

$$
1 \rightarrow S \rightarrow E \stackrel{\psi}{\rightarrow} G \rightarrow 1
$$

where $G$ is a finite group generated by $g_{i}, 1 \leq i \leq d, E$ the free product of any cyclic groups $\left\langle e_{i}\right\rangle, 1 \leq i \leq d$, and $e_{i} \psi=g_{i}$. Let $p$ be a (fixed) prime. The largest abelian $p$-quotient $\widehat{S}=S / S^{\prime} S^{p}$, regarded as an $\mathbb{F}_{p} G$-module via conjugation in $E$, is called the relative relation module (modulo $p$ ) of $G$ determined by $\psi$. If each $\left\langle e_{i}\right\rangle$ is infinite, $\widehat{S}$ is called a relation module of $G$. Gaschütz [1], Gruenberg [2, 3], and others have studied relation modules. $\widehat{S}$ is called minimal if $G$ cannot be generated by fewer than $d$ elements. As a direct consequence of [3, Theorem (2.9)], minimal relation modules of $p$-groups are nonprojective and indecomposable. The aim of this paper is to prove

Theorem 1. If $\left|\left\langle e_{i}\right\rangle\right|=m_{i}\left|\left\langle g_{i}\right\rangle\right|, 1 \leq m_{i}<\infty$, and $p+m_{i}, 1 \leq i \leq d$, then the minimal relative relation module $\widehat{S}$ of a p-group is nonprojective and indecomposable.

For the rest of the paper, let $G$ be a (finite) $p$-group and regard all modules as (right) $\mathbb{F}_{p} G$-modules. It is a well-known fact that the Frattini subgroup of $G$ coincides with $G^{\prime} G^{p}$, and hence the minimal number of generators of $G$ and $G / G^{\prime} G^{p}$ is the same. Moreover, $\mathbb{F}_{p} G$ and all its submodules are indecomposable, and $\mathbb{F}_{p} G$ has only one irreducible module, namely, $\mathbb{F}_{p}$. A minimal generating set for a module is an $\mathbb{F}_{p} G$-generating set whose cardinality is less than or equal to any other generating set for the module. For a module $M$, define $[M, G]$ to be the span of $\{m(g-1) / m \in M, g \in G\}$, so that $M /[M, G]$ is the largest trivial quotient of $M$. We set $[M, G, G]=[[M, G], G]$. The following (well-known) result is not difficult to prove.

Received by the editors November 6, 1990 .

1980 Mathematics Subject Classification (1985 Revision). Primary 16A26, 20 C05.

(C) 1993 American Mathematical Society $0002-9939 / 93 \$ 1.00+\$ .25$ per page 
Lemma 2. Let $H$ be any subgroup of $G$ and $M$ a module that affords the natural permutation representation of $G$ on the set of (right) cosets of $H$. Then

$$
[M, G] /[M, G, G] \cong G / H G^{\prime} G^{p} .
$$

Corollary 3. Let $d$ be the minimum number of generators for $G$ and $M a$ module generated by $r$ elements. Then

(a) $\operatorname{dim}(M /[M, G]) \leq r$,

(b) $\operatorname{dim}([M, G] /[M, G, G]) \leq d r$, and

(c) $\operatorname{dim}([M, G] /[M, G, G]) \leq d \operatorname{dim} M /[M, G]$.

Proof. (a) follows from the fact that the result is true for free modules of rank $r$, (b) follows by substituting $H=1$ in Lemma 2, and (c) follows from (b) by observing that the minimal number of generators for $M$ is the same as the dimension of $M /[M, G]$.

Proof of Theorem 1. From [5, (2.13)] we obtain the following $\mathbb{F}_{p} G$-exact sequence:

$$
0 \rightarrow \widehat{S} \rightarrow L \rightarrow M \rightarrow 0
$$

and

$$
0 \rightarrow M \rightarrow \bigoplus_{i=1}^{d} U_{i} \stackrel{\beta}{\rightarrow} \mathbb{F}_{p} \rightarrow 0
$$

where $L$ is a free module of rank $d-1$. Since $\widehat{S}$ is a homomorphic image of the corresponding minimal relation module that is indecomposable and nonprojective, $\widehat{S}$ has no nonzero projective direct summand. It follows that (1) is a projective cover of $M$. By a theorem of Heller [4] the indecomposability of $\widehat{S}$ will follow if we prove

Theorem. $M$ is indecomposable.

Proof. To prove this we use the following exact sequence (cf. [5, (2.13)]):

$$
0 \rightarrow M \rightarrow \bigoplus_{i=1}^{d} U_{i} \stackrel{\beta}{\rightarrow} \mathbb{F}_{p} \rightarrow 0
$$

where $U_{i}$ is the module that affords the natural permutation representation of $G$ on the cosets $\left\langle g_{i}\right\rangle$ and $u_{i} \beta=1,1 \leq i \leq d$, where $u_{i}$ is an $\mathbb{F}_{q} G$ generator of $U_{i}$. By definition of $\beta$, the kernel $M$ of $\beta$ is generated by all $u_{i}-u_{d}, 1 \leq i \leq d-1$, and hence $\operatorname{dim} M /[M, G] \leq d-1$. But $(M+[U, G]) /[U, G]$ has dimension $d-1$ and is a surjective image of $M /[M, G]$. Hence $[M, G]=[U, G] \cap M$, whence $[M, G]=[U, G]=$ $\bigoplus_{i=1}^{d}\left[U_{i}, G\right]$, and also $\operatorname{dim} M /[M, G]=d-1$. Now suppose that $M=$ $M^{\prime} \oplus M^{\prime \prime}$, and let $r=\operatorname{dim}\left(M^{\prime} /\left[M^{\prime}, G\right]\right)$. Since $[M, G]=\left[M^{\prime}, G\right] \oplus\left[M^{\prime \prime}, G\right]$ $=\bigoplus_{i=1}^{d}\left[U_{i}, G\right]$ with $\left[U_{i}, G\right]$ indecomposable, by the Krull-Schmidth theorem, $\left[M^{\prime}, G\right]$ is isomorphic to the direct sum of $s$, say, copies of $\left[U_{i}, G\right]$, and $\left[M^{\prime \prime}, G\right]$ is isomorphic to the direct sum of $r-s$ copies of $\left[U_{i}, G\right]$. By Lemma $2, \operatorname{dim}\left(\left[U_{i}, G\right] /\left[U_{i}, G, G\right]\right)=d-1$ and so

$$
\operatorname{dim}\left(\left[M^{\prime}, G\right] /\left[M^{\prime}, G, G\right]\right)=s(d-1)
$$


and

$$
\operatorname{dim}\left(\left[M^{\prime \prime}, G\right] /\left[M^{\prime \prime}, G, G\right]\right)=(d-s)(d-1) .
$$

By Corollary 3(b), however, $s(d-1) \leq d r$ and $(d-s)(d-1) \leq d(d-1-r)$. Since these two inequalities sum to an equality, both of them must be equalities. But then $d-1$ divides $r$, which is only possible when either $r=0$ or $r=d-1$. Thus either $M^{\prime}=0$ or $M^{\prime \prime}=0$, which completes the proof.

\section{ACKNOWLEDGMENT}

The author would like to thank John Cossey and Laci Kovacs, Australian National University, for providing inspiration and encouragement for the work in this paper. He would also like to thank Professor Abdus Salam, the International Atomic Energy Agency, and UNESCO for hospitality at the International Centre for Theoretical Physics, Trieste, where this work was completed.

\section{REFERENCES}

1. W. Gaschütz, Über modulare Darstellungen endlicher Gruppen, die von freien Gruppen induziert werden, Math. Z. 60 (1954), 274-286.

2. K. W. Gruenberg, Cohomological topics in group theory, Lecture Notes in Math., vol. 143, Springer-Verlag, Berlin, Heidelberg, and New York, 1970.

3. __ Relation modules of finite groups, Regional Conf. Ser. in Math., vol. 25, Amer. Math. Soc., Providence, RI, 1976.

4. A. Heller, Indecomposable representations and the loop space operation, Proc. Amer. Math. Soc. 12 (1961), 640-643.

5. M. Yamin, Relative relation modules of finite groups, Proc. Edinburgh Math. Soc. 34 (1991), 433-442.

International Atomic Energy Agency and United Nations Educational Scientific and Cultural Organization, International Centre for Theoretical Physics, Trieste, ITALY

Permanent address: Department of Mathematics, Jamia Millia Islamia, New Delhi 110025, India 